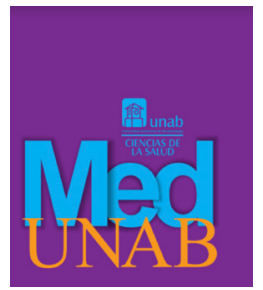

REVISTA DE LA FACULTAD

DE CIENCIAS DE LA SALUD

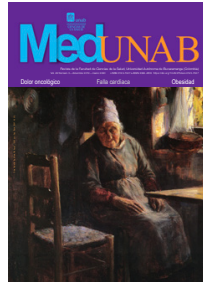

\title{
Efectividad del bloqueo neurolítico simpático abdominal en una serie de casos descriptiva en pacientes con dolor oncológico.
}

Effectiveness of neurolytic sympathetic plexus block in a descriptive case series of patients with oncological pain.

Efetividade do bloqueio neurolítico simpático abdominal em uma série descritiva de casos em pacientes com dor oncológica

Jennifer Jaimes, MD., Esp. ${ }^{1} \mathbb{D}$, Mario Andrés Leotau, MD., Esp. ${ }^{2}$ (D) German William Rangel, MD., Esp. ${ }^{2}{ }^{\mathbb{D}}$, Nelcy Miranda, Enf., Esp., MSc. ${ }^{3}$, Natalia García-Salazar. MD. ${ }^{4}$, Jully Andrea Rangel-Vera. MD. ${ }^{5}$ (D)

1. Médica Anestesióloga, Clínica FOSCAL - FOSCAL Internacional, Floridablanca, Santander, Colombia.

2. Médico, Anestesiólogo, Especialista en Medicina del Dolor y Cuidado Paliativo, Clínica de dolor Aliviar, Clínica FOSCAL-FOSCAL Internacional, Floridablanca, Santander, Colombia.

3. Enfermera, Máster en Estudio y Tratamiento del dolor, Especialista en Epidemiología, Magíster en Gerencia de Negocios, CEO - COINS Investigaciones, Bogotá, Colombia.

4. Médica, Residente de Anestesiología, Bucaramanga, Santander, Colombia.

5. Médica General, Toronto, Canadá.

Correspondencia. Jennifer Jaimes. Médico Anestesiólogo, Clínica FOSCAL, Calle 155A No. 23 - 60. Floridablanca, Santander, Colombia. Email. Ximena_9_14@hotmail.com

Cómo citar. Jaimes J, Leotau MA, Rangel GW, Miranda N, García-Salazar N, Rangel-Vera JA. Efectividad del bloqueo neurolítico simpático abdominal en una serie de casos descriptiva en pacientes con dolor oncológico. MedUNAB. 2019;22(3):330-340. doi: 10.29375/01237047.3337

\section{INFORMACIÓN DEL ARTÍCULO:}

Artículo recibido: 17 de agosto de 2018

Artículo aceptado: 15 de octubre de 2019

Doi: https://doi.org/10.29375/01237047.3337 


\section{RESUMEN}

Introducción. El dolor es el principal síntoma que se presenta en el 40\% de los pacientes oncológicos en tratamiento y en entre el $80 \%$ y el $85 \%$ de pacientes con la enfermedad avanzada. Dentro de las herramientas farmacológicas, los opioides son una opción con los consecuentes efectos secundarios, momento en el cual los procedimientos intervencionistas adquieren su importancia. El objetivo del artículo es mostrar el impacto sobre el control del dolor y la calidad de vida en paciente con dolor oncológico abdominal sometido a bloqueo celíaco o hipogástrica en un periodo de 3 meses, con el fin de generar conocimiento del tema en el área de la salud.

Metodología. Se realizó una serie descriptiva de casos en un período de 3 meses. Se incluyeron 34 pacientes adultos con dolor abdominal de origen oncológico, con propuesta de bloqueo neurolítico de plexos celíacos o hipogástrico superior como método de control del dolor y se realizó un análisis de las variables en el programa estadístico IBM SPSS Versión 19.

Resultados. Se encontró alivio del dolor en el 79.4\% de los pacientes intervenidos al poco tiempo del procedimiento y de $33.3 \%$ a los 3 meses. No se encontró significante mejoría en la calidad de vida evaluada con el cuestionario SF-36.

Conclusiones. Se encontró en este estudio que pacientes con índice de Karnofsky $<50$ tuvieron alta tasa de mortalidad posterior al bloqueo. La técnica de fenolización más radiofrecuencia podrían tener mejor respuesta terapéutica. Son necesarios más estudios para evaluar posibles asociaciones.

Palabras clave:

Dolor en Cáncer; Analgésicos Opioides; Bloqueo nervioso autónomo; Estado de ejecución de Karnofsky; Procedimientos quirúrgicos mínimamente invasivos; Calidad de vida.

\section{ABSTRACT}

Introduction. Pain is the main symptom that occurs in $40 \%$ of cancer patients undergoing treatment and between $80 \%$ and $85 \%$ of patients with advanced cancer. Out of the pharmacological tools, opioids are an option with secondary effects, which makes interventional procedures important. The objective of the article is to demonstrate the impact of celiac or hypogastric plexus block in a three-month period on the pain control and quality of life of patients with abdominal oncological pain in order to generate knowledge of this topic in the healthcare sector.

Methodology. A descriptive case series was conducted in a three-month period. Thirtyfour adult patients with oncological abdominal pain with proposed celiac or superior hypogastric plexus block as a method of pain control were included and an analysis was conducted of the variables in the statistical program IBM SPSS Version 19. Results. Pain relief was found in $79.4 \%$ of the treated patients shortly after the procedure and in $33.3 \%$ of the treated patients after 3 months. No significant improvement was evidenced in the quality of life evaluated with the SF-36 questionnaire.

Conclussions. The study found that patients with a Karnofsky performance score of $<50$ had a high mortality rate after the block. The technique of phenolization and radiofrequency could have a better therapeutic response. More studies are needed to assess possible associations.

Keywords:

Cancer pain; Opioid analgesics; Autonomic nerve block; Karnofsky performance status; Minimally invasive surgical procedures; Quality of life.

\section{RESUMO}

Introdução. A dor é o principal sintoma que ocorre em $40 \%$ dos pacientes oncológicos em tratamento e entre $80 \%$ e $85 \%$ dos pacientes com a doença avançada. Dentro 
das ferramentas farmacológicas, os opióides são uma opção com consequentes efeitos colaterais, momento em que os procedimentos intervencionistas se tornam importantes.

Objetivo. O objetivo do artigo é mostrar o impacto no controle da dor e na qualidade de vida em pacientes com dor oncológica abdominal submetido a bloqueio celíaco ou do plexo hipogástrico em um período de 3 meses, a fim de gerar conhecimento sobre o assunto na área da saúde.

Métodos. Foi feita uma série descritiva de casos durante um período de 3 meses. Foram incluídos 34 pacientes adultos com dor abdominal de origem oncológica, com proposta de bloqueio neurolítico do plexo celíaco ou hipogástrico superior como método de controle da dor e a análise das variáveis foi realizada no programa SPSS statistic 19 IBM. Resultados. Observou-se alívio da dor em 79.4\% dos pacientes operados logo após o procedimento e em 33.3\% 3 meses depois. Não houve melhora significativa na qualidade de vida avaliada com o questionário SF-36.

Discussão. Encontrou-se neste estudo que pacientes com índice de Karnofsky $>50$ apresentaram alta taxa de mortalidade após o bloqueio. A técnica de fenolização e a radiofrequência poderiam ter melhor resposta terapêutica. Mais estudos são necessários para avaliar possíveis associações.

Palavras-chave:

Dor do Câncer; Analgésicos Opióides; Bloqueio nervoso autônomo; Avaliação de Estado de Karnofsky; Procedimentos cirúrgicos minimamente invasivos; Qualidade de vida.

\section{Introducción}

El dolor es el principal síntoma en el $40 \%$ de los pacientes oncológicos en tratamiento y en entre el $80 \%$ y el $85 \%$ de pacientes con la enfermedad avanzada (1). El alivio del dolor y los cuidados paliativos son prioritarios en el programa mundial del cáncer de la Organización Mundial de la Salud (OMS) (2), sabiendo que en el mundo hoy en día existen más de 37 millones de personas con este padecimiento (3). El cáncer ha llegado a tener una incidencia hasta de 10 millones de casos en América (4), incrementándose día a día hasta convertirse en un problema de salud pública. Dicha patología puede generar deterioro en la calidad de vida del paciente y limitación en sus actividades diarias.

La incidencia del dolor oncológico depende del tipo de tumor: óseo $(85 \%)$, genitourinario $(66 \%)$, de mama (34\%), de pulmón (69\%), gastrointestinal (34\%), en el páncreas $(17 \%)$, nódulos linfáticos, en el sistema hematopoyético $(5 \%)$ o en el hígado (12\%), entre otros. La incidencia de dolor oncológico abdominal, comparado con otras regiones corporales como sistema hematopoyético es del $27 \%(4,5)$.

Dentro de las herramientas farmacológicas, se proponen una variedad de fármacos tales como el metimazol, el paracetamol; coadyuvantes analgésicos como los anticonvulsivantes; antidepresivos y bifosfonatos, usados en asociación a los analgésicos en caso de predominar el componente neuropático $(6,7)$. Medicamentos como los corticoides se usan gracias a su acción antiinflamatoria, indicándose en especial para el dolor oncológico debido a metástasis óseas, hipertensión intracraneal, tumores de cara o cuello, compresión medular o nerviosa y hepatomegalia $(8,9)$; pero el pilar de manejo del dolor oncológico lo encabezan los opioides, comparándose con su estándar que es la morfina $(10,11)$, con una gran eficacia pero con efectos adversos tales como sequedad de boca en un $39 \%$, somnolencia en un $38 \%$, estreñimiento en un $35 \%$ y náuseas y vómito en un $22 \%$ (12). Sin embargo, en casos de dolor refractario, la aplicación de anestésicos locales y agentes neurolíticos en diversos nervios y ganglios se considera una opción acertada, con el advenimiento de nuevos tratamientos analgésicos como fármacos, sistemas implantables de liberación de medicamentos y técnicas neuroquirúrgicas $(13,14)$.

Un bloqueo se define como la interrupción de la transmisión nerviosa por un periodo de tiempo, y en ocasiones permanente (simpatectomía química). Los bloqueos se pueden realizar con fines diagnósticos, pronósticos o terapéuticos mediante la administración de fármacos de acción prolongada tales como sustancias neurolíticas, corticoides o anestésicos locales según la clínica del paciente $(15,16)$. El bloqueo neurolítico consiste en la destrucción de forma temporal o transitoria de la transmisión nerviosa de las fibras de los nervios con diferentes sustancias, entre ellas alcohol 
y fenol. La literatura consultada documenta la eficacia de las técnicas de bloqueo neurolítico en cuanto a la reducción de la intensidad del dolor, (16), así como la ventaja de servir como coadyuvante para reducir la dosis de opioides y sus efectos adversos (18). Los bloqueos neurolíticos tienen un papel muy importante como parte de una estrategia terapéutica (19). De igual manera, cuando se realizan con radiofrecuencia presentan resultados óptimos (20).

Dentro de los bloqueos neurolíticos para el manejo del dolor oncológico abdominal se encuentra el bloqueo del plexo celíaco y el bloqueo del plexo hipogástrico superior (20). La ubicación del bloqueo fue determinada por el médico anestesiólogo especialista en dolor según la ubicación de lesión o infiltración a nivel abdominal. En caso de encontrarse a nivel abdominal alto se procedía a realizar bloqueo celíaco; si se determinaban comprometidas las vísceras abdominales inferiores se realizaba un bloqueo hipogástrico, y finalmente, si se comprometían a nivel superior e inferior se realizaban los dos bloqueos (12).

El objetivo del artículo es mostrar el impacto sobre el control del dolor y la calidad de vida en pacientes con dolor oncológico abdominal sometidos a bloqueo celíaco o hipogástrico en un periodo de 3 meses con el fin de generar conocimiento en el medio de la salud sobre el tema.

\section{Metodología}

Se realizó un estudio descriptivo de una serie de casos captados de julio del 2014 a octubre del 2015 en un período de 3 meses cuya población fueron pacientes adultos con dolor abdominal de origen oncológico con propuesta de manejo terapéutico de bloqueo neurolítico de plexos celíaco o hipogástrico superior como método para controlar el dolor, en una clínica de cuarto nivel de complejidad en Floridablanca, Santander.

Se captaron los pacientes que cumplieran como criterios de inclusión los de ser mayores de 18 años y contar con un diagnóstico de cáncer de origen abdominal primario o metastásico a cavidad abdominal. Como criterios de exclusión se tomaron pacientes con dolor abdominal crónico de origen no oncológico previo al procedimiento, capacidad mental alterada, pacientes embarazadas, pacientes programados para manejo quirúrgico durante el seguimiento del estudio, pacientes con referencia de otra fuente de dolor diferente a la región abdominal, pacientes que hubiesen tenido radioterapia durante los 30 días previos al procedimiento en cavidad abdominal $\mathrm{y}$, pacientes con antecedentes documentados en la historia clínica de consumo de sustancias psicoactivas ilícitas en el último año.

El trabajo operativo se llevó a cabo por un grupo integral constituido por médicos generales, psicólogas, enfermeras e investigadores del proyecto. Se diligenció un formulario de captación y consentimiento informado previo a realización del procedimiento donde se tomaron variables como edad, sexo, peso, diagnóstico, estadio del cáncer, índice de Karnofsky (IK), tiempo de evolución del dolor abdominal, intensidad del dolor medida en escala numérica de dolor (NRS), radioterapia, quimioterapia y consumo de opioides. Adicionalmente se diligencia la escala 36- Item Short Form Health Survey (SF 36), que es un instrumento de medición de la calidad de vida con validación en español, el cual se realizó en el primer contacto con paciente. Observación a las 24 horas, 48 horas, 72 horas, al mes y a los 3 meses después del procedimiento por medio de llamada telefónica para evaluar intensidad de dolor en NRS, requerimiento de opioides, efectos secundarios y características del dolor. Igualmente, en la llamada del mes se repite la escala SF 36.

Se realizó un Consentimiento Informado autorizado por el Comité de Ética Médica de la clínica y de la universidad que los participantes firmaron antes de ingresar al estudio y ser sometidos a intervención. Este documento tiene la finalidad del estudio y la autorización para tomar datos de la historia clínica.

\section{Análisis estadístico}

Se analizó un grupo de 34 pacientes con diagnóstico de dolor abdominal de origen oncológico, sometidos a bloqueo neurolítico celíaco o hipogástrico. Análisis de las características de la población, alivio del dolor posterior al procedimiento hasta los 3 meses, cuantificación de requerimiento opioide posterior al procedimiento e impacto en la calidad de vida. Se utilizó IBM SPSS Versión 19 para análisis de las variables.

\section{Resultados}

Durante el periodo de estudio se identificaron 40 pacientes como posibles candidatos para inclusión en el estudio. 6 de ellos cumplían con criterio de exclusión por lo que se ingresaron en total 34 pacientes al estudio (Figura 1). De los pacientes incluidos, el 61.8\% fueron mujeres. La media de edad y peso fue 57.2 con una desviación estándar (DS) de \pm 16.2 años y $61.5 \mathrm{~kg}$ 


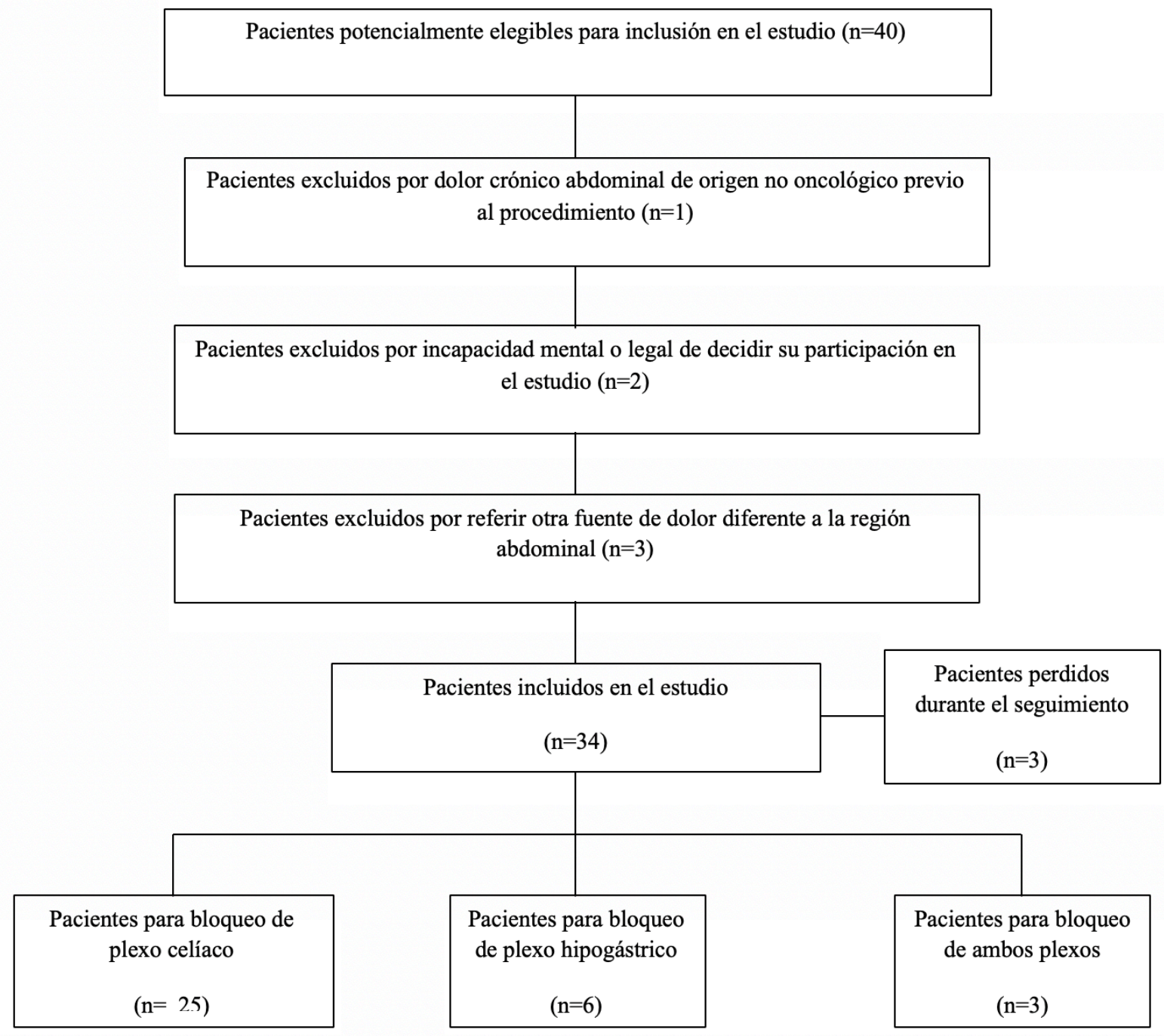

Figura 1. Selección de pacientes incluidos en el estudio

$(\mathrm{DS} \pm 7,4)$ respectivamente. Dentro de las patologías más frecuentes se encontró el adenocarcinoma gástrico en un $41.2 \%$, seguido del carcinoma de páncreas en un $17.6 \%$. Los pacientes incluidos tenían una clasificación en la escala TNM (tamaño, nódulo, metástasis) del $64,7 \%$ en T4. El $47,1 \%$ fueron N2 y el $82.4 \%$ M1. El IK fue menor o igual a 50 en el $47.1 \%$ de los casos y el $73.5 \%$ se encontraban en manejo intrahospitalario.

La media de dolor fue de 6,8 (DS $\pm 1,2)$ previo al procedimiento y el $55,9 \%$ presentaba dolor agudo. Se registró una media de consumo de opioide previo a procedimiento de $16.3 \mathrm{mg}$ de morfina (DS $\pm 11,6)$ y el $85.3 \%$ reportó consumo de neuromoduladores previo al mismo. Se realizó bloqueo celíaco en el $73.5 \%$ de los pacientes, bloqueo hipogástrico al $17.6 \%$ y los dos bloqueos al 8.8\%. En relación con el tipo de bloqueo realizado en el $73.5 \%$, se realizó fenolización (uso de fenol en el bloqueo). En el 11.8\% se realizó fenolización más radiofrecuencia y solo bloqueo con analgésico local en el $14.7 \%$ de los pacientes. En cuanto a los medicamentos usados durante el procedimiento, la mezcla más usada fue triamcinolona, dexametasona, ketamina, morfina, bupivacaína y fenol en un $76.5 \%$ de los pacientes intervenidos.

Se encontró que el $79.4 \%$ de los pacientes intervenidos refirieron alivio del dolor inmediatamente después del procedimiento; el $72.72 \%$ refirió sentir persistencia de alivio a las 24 horas siguientes; el $50 \%$ a las 48 horas; el $43.44 \%$ a las 72 horas; el $66.6 \%$ reportó alivio al mes; y el $33.3 \%$ a los tres meses (Tabla 1). Por otra parte, se evidenció una baja en el consumo de morfina solo durante las primeras 72 horas con posterior incremento 
Tabla 1. Pacientes con alivio del dolor a través del estudio según ubicación del bloqueo.

\begin{tabular}{lllll}
\hline CONTROL DEL DOLOR & $\begin{array}{l}\text { Plexo celíaco } \\
\mathbf{n}\end{array}$ & $\begin{array}{l}\text { Plexo hipogástrico } \\
\mathbf{n}\end{array}$ & $\begin{array}{l}\text { Ambos } \\
\mathbf{n}\end{array}$ & $\begin{array}{l}\text { Total } \\
\mathbf{\%}\end{array}$ \\
\hline Posterior a procedimiento $(\mathbf{n}=\mathbf{3 4})$ & 20 & 4 & 3 & $\frac{27}{79.4}$ \\
\hline $\mathbf{2 4}$ horas $(\mathbf{n}=\mathbf{3 3})$ & 19 & 3 & 2 & $\frac{24}{72.72}$ \\
\hline $\mathbf{4 8}$ horas $(\mathbf{n}=\mathbf{3 2})$ & 12 & 2 & 2 & 16 \\
\hline $\mathbf{7 2}$ horas $(\mathbf{n}=\mathbf{3 0})$ & 10 & 2 & 1 & 50 \\
\hline Mes $(\mathbf{n}=\mathbf{1 8})$ & 9 & 1 & 2 & 13 \\
\hline Tres meses $(\mathbf{n}=\mathbf{1 2})$ & 3 & 0 & 1 & 43.33 \\
\hline
\end{tabular}

Fuente: Tabla elaborada por los autores.

Tabla 2. Requerimiento de morfina durante el periodo de seguimiento según ubicación del bloqueo.

\begin{tabular}{|c|c|c|c|c|}
\hline Variable & $\begin{array}{l}\text { Plexo celíaco } \\
\text { Media, } \pm \text { DS } \\
\text { Mediana, } \mathbf{m g}\end{array}$ & $\begin{array}{l}\text { Plexo hipogástrico } \\
\text { Media, } \pm \text { DS } \\
\text { Mediana, mg }\end{array}$ & $\begin{array}{l}\text { Ambos } \\
\text { Media, } \pm \text { DS } \\
\text { Mediana, mg }\end{array}$ & $\begin{array}{l}\text { Total } \\
\text { Media, } \pm \text { DS } \\
\text { Mediana; } \mathbf{m g}\end{array}$ \\
\hline $\begin{array}{l}\text { Previo procedimiento } \\
(\mathrm{n}=)\end{array}$ & $\begin{array}{l}17.9 \pm 6.11 \\
12\end{array}$ & $\begin{array}{l}11.17 \pm 10,2 \\
11\end{array}$ & $\begin{array}{l}13.3 \pm 6,11 \\
12\end{array}$ & $\begin{array}{l}16.30 \pm 11.67 \\
12\end{array}$ \\
\hline $\begin{array}{l}\text { Posterior a procedimiento } \\
24 \text { horas }(n=)\end{array}$ & $\begin{array}{l}8.76 \pm 7.64 \\
8\end{array}$ & $\begin{array}{l}5 \pm 4.15 \\
4\end{array}$ & $\begin{array}{l}6 \pm 5.66 \\
6\end{array}$ & $\begin{array}{l}7.90 \pm 7.06 \\
6,00\end{array}$ \\
\hline 48 horas $(n=)$ & $\begin{array}{l}12 \pm 12.39 \\
10\end{array}$ & $\begin{array}{l}10.67 \pm 7.23 \\
10\end{array}$ & $\begin{array}{l}10 \pm 11.31 \\
10\end{array}$ & $\begin{array}{l}11,62 \pm 11.26 \\
10,00\end{array}$ \\
\hline 72 horas $(n=)$ & $\begin{array}{l}8.41 \pm 6.53 \\
8\end{array}$ & $\begin{array}{l}10.83 \pm 10.34 \\
6,5\end{array}$ & $\begin{array}{l}16.5 \pm 19 \\
16,5\end{array}$ & $\begin{array}{l}9.43 \pm 8.15 \\
8,00\end{array}$ \\
\hline $\operatorname{Mes}(n=)$ & $\begin{array}{l}62.33 \pm 60.43 \\
40\end{array}$ & $\begin{array}{l}14 \pm 0 \\
14\end{array}$ & $\begin{array}{l}10 \pm 14.14 \\
10\end{array}$ & $\begin{array}{l}53.83 \pm 58.32 \\
20\end{array}$ \\
\hline Tres meses $(n=)$ & $\begin{array}{l}198 \pm 339.3 \\
100\end{array}$ & - & $\begin{array}{l}35 \pm 0 \\
35\end{array}$ & $\begin{array}{l}184.75 \pm 327.01 \\
80\end{array}$ \\
\hline
\end{tabular}

Fuente: Tabla elaborada por los autores. 
importante de las nuevas dosis con respecto a las dosis basales, al mes y a los tres meses (Tabla 2).

Con respecto a los efectos secundarios, se encontró que el $94.1 \%$ de los pacientes sometidos a procedimientos intervencionistas no presentaron eventos adversos mayores, el $2.9 \%$ presentó choque distributivo y otro $2.9 \%$ presentó paraplejia. En el $100 \%$ de los casos de choque y paraplejia, se dio en el grupo de pacientes a los que se les realizó bloqueo de plexo celíaco. En cuanto a los efectos secundarios menores, el $47.05 \%$ del total de los pacientes no presentó ningún efecto secundario, el $8 \%$ presentó enfermedad diarreica, $14.7 \%$ hipotensión y el $29.4 \%$ presentó vómito. Dichos efectos secundarios menores fueron más frecuentes en el bloqueo de plexo celíaco.

Durante el seguimiento fallecieron el $67.7 \%$ de los pacientes. Del total de fallecidos el $66.7 \%$ correspondía a pacientes sometidos a bloqueo de plexo celíaco, $23,8 \%$ a bloqueo hipogástrico y el $9.5 \%$ a bloqueo de plexo celíaco e hipogástrico a la vez. El tiempo promedio transcurrido entre la realización del procedimiento y el fallecimiento fue de $21.48 \pm 25.81$ días. Se encontró que el mayor porcentaje de pacientes fallecidos estaban hospitalizados y todos los pacientes con IK de entre 0 y 50 fallecieron. Se aumentó 4 veces el riesgo de mortalidad en estos pacientes; con IK mayor de 50 cuando se realiza bloqueo celíaco. En el estudio de Guerrero et al. se evalúa la supervivencia de los pacientes con tumores de origen gastrointestinal y se encuentra que los pacientes con IK entre 0 y 50 tienen mayor riesgo de mortalidad.

En cuanto al cuestionario de calidad de vida SF-36 en sus 8 dimensiones se encontró un valor de $56.8 \%$ (DS \pm 14.4$)$ para funcionamiento físico previo a la intervención, de $58.45 \%$ (DS \pm 16.20 ) para limitación secundaria a problemas físicos de $80.87 \%$ (DS \pm 14.60 ) para dolor corporal, de $59.41 \%(\mathrm{DS} \pm 14.55)$ para funcionamiento o rol social, de $60.65 \%$ (DS \pm 8.90 ) en salud mental previa, de $60.78 \%$ (DS \pm 17.35 ) en limitación por problemas emocionales, de $59.27 \%$ (DS \pm 9.90 ) en vitalidad, energía y fatiga y de $70,97 \%$ (DS \pm 10.44 ) en percepción de la salud. Las mediciones, que fueron realizadas a las 24 horas, a las 72 horas, al mes y a los tres meses, se encuentran detalladas en la tabla 3.

Con relación al impacto en la calidad de vida de los pacientes, se estableció la diferencia de medias entre las dimensiones del cuestionario SF36 previo y el control realizado al mes y a los tres meses, se encontró una disminución estadísticamente significativa de $14.07 \%$ ( $p$ 0.01) en la dimensión de funcionamiento físico y una reducción del 15.91\% (p 0.04) en la dimensión limitaciones secundarias a problemas físicos, al establecer la diferencia entre la evaluación previa y la realizada a los tres meses (Tabla 3).

\section{Discusión}

El dolor es considerado un síntoma principal en el $40 \%$ de los pacientes oncológicos de etiología abdominal. $\mathrm{Su}$ control se convierte en un aspecto importante en la conservación de la calidad de vida de estos pacientes. También ha relacionado con el aumento de la expectativa de vida. En muchas ocasiones el dolor se torna tan severo que, desafortunadamente, el manejo con opioides resulta limitado, llegando a tener gran cantidad de efectos adversos.

Con relación al alivio del dolor, se evidenció mejoría en la mayoría de los pacientes llevados a procedimiento. Dicho alivio disminuye a lo largo del tiempo, lo cual se correlaciona con lo descrito en la literatura, en donde se manifiesta que con el bloqueo neurolítico se obtiene un alivio de dolor, pero que este no supera los seis meses $(21,22)$. Cuando se trata de establecer qué variables están relacionadas con dicho alivio, se encontró que los pacientes con pesos entre 61 y $70 \mathrm{~kg}$ tienen más posibilidades de aliviarse después de la realización del procedimiento del bloqueo neurolítico que los pacientes en otros rangos de peso. Este hallazgo no había sido reportado en estudios previos.

El alivio del dolor podría estar directamente relacionado con el consumo de opioides ya que un alto porcentaje de pacientes los requirieron durante las 24, 48 y 72 horas posteriores al procedimiento, así como al mes y a los tres meses. Un puntaje alto en el IK también podría estar relacionado con el alivio, pues se observó un mayor alivio en los pacientes con $\mathrm{IK}>60$ independientemente del tipo de bloqueo realizado.

El tipo de bloqueo también es determinante en el alivio del dolor: se observó más pacientes con alivio de dolor en los que se les realizó fenolización más radiofrecuencia en el grupo de bloqueo de plexo celíaco. Sin embargo, el porcentaje de pacientes que refirió alivio del dolor en este grupo fue similar, tanto para fenolización como para solo bloqueo, siendo estos muchos menores que el reportado con fenolización más radiofrecuencia. Al contrario, en el bloqueo de plexo hipogástrico se encontró un menor porcentaje de pacientes con alivio de dolor cuando se realiza radiofrecuencia más fenolización o solo fenolización (siendo estos similares). Este hallazgo 
Tabla 3. Mediciones de la escala SF36 a lo largo del estudio.

\begin{tabular}{|c|c|c|c|c|c|c|}
\hline Dimensiones & $\mathbf{N}$ & Media & DS & Varianza & $\mathbf{z}$ & $\mathbf{p}$ \\
\hline \multicolumn{7}{|l|}{ Funcionamiento físico } \\
\hline Previo & 34 & 56.85 & 14.65 & 214.6 & - & - \\
\hline $\mathrm{Al} \mathrm{mes}$ & 18 & 62.96 & 19.1 & 364.69 & - & - \\
\hline A los tres meses & 11 & 43.12 & 20.34 & 413.67 & - & - \\
\hline Diferencia al mes - previo & 18 & 5.12 & 19.03 & 362.05 & -1.24 & 0.21 \\
\hline Diferencia a los tres meses - previo & 11 & -14.07 & 20.3 & 411.97 & -2.39 & 0.01 \\
\hline \multicolumn{7}{|c|}{ Limitaciones secundarias a problemas físicos } \\
\hline Previo & 34 & 58.46 & 16.2 & 262.51 & - & - \\
\hline $\mathrm{Al}$ mes & 18 & 59.58 & 17.66 & 311.95 & - & - \\
\hline A los tres meses & 11 & 50 & 0 & 0 & - & - \\
\hline Diferencia al mes - previo & 18 & -4.31 & 19.46 & 378.53 & -0.84 & 0.4 \\
\hline Diferencia a los tres meses - previo & 11 & -15.91 & 20.98 & 440.34 & -2.03 & 0.04 \\
\hline \multicolumn{7}{|l|}{ Dolor corporal } \\
\hline Previo & 34 & 80.88 & 14.6 & 213.24 & - & - \\
\hline $\mathrm{Al}$ mes & 18 & 70.09 & 18.8 & 353.29 & - & - \\
\hline A los tres meses & 11 & 71.36 & 8.63 & 74.4 & - & - \\
\hline Diferencia al mes - previo & 18 & -8.34 & 22.87 & 522.98 & -1.68 & 0.09 \\
\hline Diferencia a los tres meses - previo & 11 & -8.64 & 15.35 & 235.64 & -1.38 & 0.16 \\
\hline \multicolumn{7}{|l|}{ Funcionamiento o rol social } \\
\hline Previo & 34 & 59.41 & 14.55 & 211.77 & - & - \\
\hline Al mes & 18 & 60.74 & 15.77 & 248.78 & - & - \\
\hline A los tres meses & 11 & 62.55 & 10.32 & 106.47 & - & - \\
\hline Diferencia al mes - previo & 18 & 1.85 & 20.57 & 423 & -1.39 & 0.16 \\
\hline Diferencia a los tres meses - previo & 11 & 7.09 & 15.11 & 228.29 & -1.42 & 0.15 \\
\hline \multicolumn{7}{|l|}{ Salud mental } \\
\hline Previo & 34 & 60.66 & 8.9 & 79.22 & - & - \\
\hline Al mes & 18 & 56.44 & 11.63 & 135.32 & - & - \\
\hline A los tres meses & 11 & 59.09 & 9.44 & 89.09 & - & - \\
\hline Diferencia al mes - previo & 18 & -4.62 & 13.6 & 184.96 & -1.22 & 0.22 \\
\hline Diferencia a los tres meses - previo & 11 & 0.08 & 13.15 & 172.84 & -0.51 & 0.95 \\
\hline \multicolumn{7}{|c|}{ Limitación por problemas emocionales } \\
\hline Previo & 34 & 60.78 & 17.35 & 301.03 & - & - \\
\hline $\mathrm{Al}$ mes & 18 & 58.52 & 9.3 & 86.57 & - & - \\
\hline A los tres meses & 11 & 60.61 & 4.43 & 19.6 & - & - \\
\hline Diferencia al mes - previo & 18 & -7.22 & 23.16 & 536.28 & -0.92 & 0.35 \\
\hline Diferencia a los tres meses - previo & 11 & -9.09 & 22.42 & 502.45 & -1.07 & 028 \\
\hline \multicolumn{7}{|l|}{ Vitalidad, energía o fatiga } \\
\hline Previo & 34 & 59.27 & 9.9 & 98.06 & - & - \\
\hline $\mathrm{Al}$ mes & 18 & 56.85 & 16.19 & 262.08 & - & - \\
\hline A los tres meses & 11 & 57.58 & 11.46 & 131.31 & - & - \\
\hline Diferencia al mes - previo & 18 & 0.14 & 17.64 & 311.12 & -1.14 & 0.25 \\
\hline Diferencia a los tres meses - previo & 11 & 1.52 & 12.95 & 167.61 & 0.00 & 1.00 \\
\hline \multicolumn{7}{|l|}{ Percepción de la salud } \\
\hline Previo & 34 & 70.97 & 10.44 & 109 & - & - \\
\hline Al mes & 18 & 59.03 & 8.72 & 76.07 & - & - \\
\hline A los tres meses & 11 & 57.58 & 6.41 & 41.04 & - & - \\
\hline Diferencia al mes - previo & 18 & -11.64 & 16.36 & 267.74 & -2.46 & 0.01 \\
\hline Diferencia a los tres meses - previo & 11 & -9.7 & 13.65 & 186.22 & -1.86 & 0.06 \\
\hline
\end{tabular}

Fuente: Tabla elaborada por los autores. 
hasta el momento no parece haber sido reportado antes en la literatura.

En el estudio se evidenció un incremento progresivo en el consumo de opioides con el paso del tiempo con una disminución del consumo solo durante las primeras 72 horas después del procedimiento, con posterior incremento de las dosis con respecto a las dosis basales al mes y a los tres meses. Estudios previos reportan que la realización del bloqueo puede estar directamente relacionada con el alivio del dolor, pero no con la suspensión de la terapia opioide. Sin embargo, también manifiestan que puede haber una disminución en el consumo de opioides y en los efectos secundarios de los mismos $(22,23)$.

Se encontró que el mayor porcentaje de pacientes fallecidos estaban hospitalizados y que todos los pacientes con IK de 0 a 50 puntos fallecieron, con un aumento de 4 veces el riesgo de mortalidad, comparándose con los que tienen un índice mayor de 50 cuando se realiza bloqueo celíaco. Guerrero et al, en un estudio en el que se evalúa la supervivencia de los pacientes con tumores de origen gastrointestinal, encuentran que los pacientes con IK entre 0 y 50 , tienen mayor riesgo de mortalidad (24).

Dentro de los eventos adversos, el choque y la paraplejia se presentaron en un $2.9 \%$ respectivamente, lo cual corresponde a lo reportado en la literatura por Teixeria et al, con una frecuencia del 1 al 2\% (25-27). Los pacientes que tomaban neuromoduladores previos al procedimiento no presentaron ningún efecto secundario. Este hallazgo no está reportado en la literatura.

Con relación a los efectos secundarios menores, el vómito fue más frecuente con un $29.4 \%$, seguido de hipotensión en $14.7 \%$ y enfermedad diarreica en $8 \%$; lo cual se correlaciona con lo reportado por Teixeria et al: vómito en un $40 \%$, hipotensión del $4.3 \%$ al $8 \%$ y deposiciones diarreicas en un 3.2\% (27). En el presente estudio se encontró que $67.7 \%$ de los pacientes falleció durante el periodo de seguimiento siendo mucho mayor este porcentaje en el grupo llevado a bloqueo de plexo celíaco. Este es el grupo de bloqueo neurolítico más frecuente, y sus integrantes presentaron una tasa de sobrevida de tan solo 21 días posteriores al procedimiento. Teniendo en cuenta que el mayor porcentaje de pacientes incluidos en este estudio presentaron un diagnóstico de adenocarcinoma gástrico, seguido por cáncer de páncreas, se evidencia en la literatura una sobrevida para los pacientes con cáncer gástrico metastásico con afectación ganglionar del $20 \%$ al $30 \%$ de hasta cinco años y para los pacientes con cáncer de páncreas se reportan hasta 6 meses $(27,28)$.

Al evaluar el impacto posterior en la calidad de vida que tiene el procedimiento, se observa que para el total de los pacientes hay un empeoramiento, desde limitaciones secundarias hasta problemas físicos importantes (que se evidencian a los tres meses). También empeoran la percepción de la salud; cosa que se observa al mes. Con respecto a la ubicación del procedimiento, dichos empeoramientos se observan en el grupo al que se le realizó bloqueo de plexo celíaco y se observó empeoramiento en la dimensión de dolor corporal en este mismo grupo. En la dimensión de funcionamiento o rol social se observa una mejoría al mes después el procedimiento.

En la valoración de la calidad de vida en los pacientes en los cuales se realiza bloqueo neurolítico, no se ha utilizado el instrumento SF-36. Sin embargo, sí se han usado otros instrumentos como el Quality of Life (QoL) (20-31), en los cuales se ha evidenciado una mejoría leve de los índices la calidad de vida con seguimientos a 60 días.

\section{Conclusiones}

Los bloqueos neurolíticos son útiles para los pacientes con dolor abdominal secundario a patología oncológica, ya que proveen alivio del dolor, por lo menos en el primer mes posterior al procedimiento. Este no es un reemplazo a la terapia con opioides, sino que forma parte del manejo multimodal de los pacientes.

En el estudio, los pacientes con $\mathrm{IK}<50$ tuvieron mayor desenlace fatal después del bloqueo neurolítico, lo cual abre las puertas a posibles estudios específicos que evalúen esta asociación bajo un modelo multivariado. Realizar fenolización más radiofrecuencia podría tener mejor respuesta terapéutica, por lo que también son necesarios estudios más grandes para evaluar esta asociación.

Se deben evaluar los riesgos y beneficios de la realización del bloqueo neurolítico, teniendo en cuenta los posibles eventos adversos, efectos secundarios y frecuencia de mortalidad, señalados en este artículo. Se recomienda hacer estudios analíticos en los cuales se pueda evaluar, con un mayor tamaño de la muestra, las consecuencias de los bloqueos neurolíticos simpáticos abdominales. 


\section{Financiación}

Los autores declaran no haber recibido patrocinio económico para la realización del proyecto

\section{Referencias}

1. Van Hecke O, Torrance N, Smith BH. Chronic pain epidemiology and its clinical relevance. Br J Anaesth. 2013 Jul;111(1):13-8. doi: 10.1093/bja/aet123.

2. Organización Mundial de la Salud. Programas nacionales de control del cáncer políticas y pautas para la gestión; 2004. Recuperado a partir de: https:/www. paho.org/hq/dmdocuments/2012/OPS-ProgramasNacionales-Cancer-2004-Esp.pdf

3. Bruera E, Kim HN. Cancer Pain. JAMA. 2003;290(18):2476-2479. doi:10.1001/ jama.290.18.2476

4. Auret K, Schug SA. Best Pract Res Clin Anaesthesiol. 2013 Dec;27(4):545-61. doi: 10.1016/j. bpa.2013.10.007. Epub 2013 Oct 15.

5. Gangi A, Buy X, Garnon J, Tsoumakidou G, Moser $\mathrm{T}$, Bierry $\mathrm{G}$, et al. Pain management in oncology. J Radiol 2011 Sep;92(9):801-813. DOI: 10.1016/j. jradio.2011.07.014

6. Prieto Ríos B, Navarro Expósito F, Molina Villaverde R, Álvarez-Mon Soto M. Tratamiento del dolor oncológico. Medicine-Programa de Formación Médica Continuada Acreditado 2009;10(24):16291633. https://doi.org/10.1016/S0211-3449(09)700104

7. Sancho JF, López G, Sancho A, Ávila C, Grande ML, Cavallo L, et al. Tratamiento del dolor oncológico. Psicooncología 2006;3(1):121-138. Recuperado a partir de: https://revistas.ucm.es/index.php/PSIC/ article/view/PSIC0606130121A/15941

8. Goodman Gilman A. Las bases farmacológicas de la terapéutica, décima edición. México, editorial Mc Graw Hill Interamericana 2003;2:104-106. Recuperado a partir de: https://dvmbooks.weebly. com/uploads/2/2/3/6/22365786/2._goodman_and_ gilman.pdf

9. De Leon-Casasola OA. Current developments in opioid therapy for management of cancer pain. Clin J Pain 2008 May;24 Suppl 10:S3-7. doi: 10.1097/ AJP.0b013e31816b589f.

10. Mearis M, Shega JW, Knoebel RW. Does Adherence to National Comprehensive Cancer Network Guidelines Improve Pain-Related Outcomes? An Evaluation of Inpatient Cancer Pain Management at an Academic Medical Center. J Pain Symptom Manage. 2014 Sep;48(3):451-8. https://doi.org/10.1016/j. jpainsymman.2013.09.016
11. Celis-Rodriguez E, Birchenall $\mathrm{C}$, de la Cal MA, Castorena Arellano G, Hernandez A, Ceraso D, Díaz Cortés JC, Dueñas Castell C, Jimenez EJ, Meza JC, Muñoz Martínez T, Sosa García JO, Pacheco Tovar C, Pálizas F, Pardo Oviedo JM, Pinilla DI, Raffán-Sanabria F, Raimondi N, Righy Shinotsuka C, Suárez M, Ugarte S, Rubiano S. Clinical practice guidelines for evidence-based management of sedoanalgesia in critically ill adult patients. Med Intensiva 2013 Nov;37(8):519-574. doi: 10.1016/j. medin.2013.04.001

12. Pernia A, Torres L. Tratamiento del dolor oncológico terminal intenso. Revista de la Sociedad Española del Dolor 2008;15(5):308-324. Recuperado a partir de: $\quad$ http://scielo.isciii.es/scielo.php?script=sci arttext\&pid=S1134-80462008000500005\#back

13. De Andres J, Asensio-Samper JM, Fabregat-Cid G. Intrathecal delivery of analgesics. Methods Mol Biol. 2014;1141:249-78. doi: 10.1007/978-1-4939-0363416

14. Brogan S, Junkins S. Interventional therapies for the management of cancer pain. J Support Oncol. 2010 Mar-Apr;8(2):52-9.

15. Birthi P, Sloan P. Interventional treatment of refractory cancer pain. Cancer J. 2013 Sep-Oct;19(5):390-6. doi: 10.1097/PPO.0b013e3182a631a2.

16. Nelson R. New nerve-block analgesia for patients with pancreatic cancer. Lancet Oncol 2004 Apr;5(4):201. DOI:https://doi.org/10.1016/S1470-2045(04)01442-1

17. Cepeda MS, Farrar JT. Economic evaluation of oral treatments for neuropathic pain. J Pain. 2006 Feb;7(2):119-28. DOI: 10.1016/j.jpain.2005.09.004

18. Delaplace M, Maitre F, Dufour T, Landrieux I, Gauthier P, Jebira P, et al. Two cases of cutaneous erythema following spinal cord stimulation]. Ann Dermatol Venereol. 2010 Apr;137(4):297-300. doi: 10.1016/j.annder.2010.02.017.

19. Cruccu G, Aziz T, Garcia $\square$ Larrea L, Hansson P, Jensen T, Lefaucheur $J$, et al. EFNS guidelines on neurostimulation therapy for neuropathic pain. European Journal of Neurology 2007;14(9):952-970. DOI: 10.1111/j.1468-1331.2007.01916.x

20. Brogan S, Junkins S. Interventional therapies for the management of cancer pain. J Support Oncol 2010 Mar-Apr;8(2):52-59. Recuperado a partir de: https:// www.ncbi.nlm.nih.gov/pubmed/20464881

21. Marcy PY, Magne N, Descamps B. Coeliac plexus block: utility of the anterior approach and the real time colour ultrasound guidance in cancer patient. Eur J Surg Oncol 2001 Dec;27(8):746-749. DOI: 10.1053/ ejso.2001.1202

22. Staats PS, Hekmat H, Sauter P, Lillemoe K. The effects of alcohol celiac plexus block, pain, and mood on longevity in patients with unresectable pancreatic 
cancer: a double-blind, randomized, placebocontrolled study. Pain Med. 2001 Mar;2(1):28-34. DOI: 10.1046/j.1526-4637.2001.002001028.x

23. Bhatnagar S, Gupta M. Evidence-based Clinical Practice Guidelines for Interventional Pain Management in Cancer Pain. Indian J Palliat Care. 2015 May-Aug;21(2):137-47. doi: 10.4103/09731075.156466.

24. Guerrero M: Gómez A; Pérez S; Gómez A; Pedraza A. Correlación del estado funcional de Karnofsky con la supervivencia de pacientes con tumores de origen gastrointestinal en un servicio de cuidados paliativos. Asociación Mexicana de Cirugía General. Elseiver. 2014. Recuperado a partir de: https://www.elsevier.es/es-revista-cirujano-general218-articulo-correlacion-del-estado-funcionalkarnofsky-X1405009914734080

25. Adradra J; Calambas F; Díaz J. Características sociodemográficas y clínicas en una población con cáncer gástrico en el Cauca, Colombia. 2008. Asociaciones Colombianas de Gastroenterología, Endoscopia digestiva, Coloproctología y Hepatología. Recuperado a partir de: https://www.redalyc.org/ pdf/3377/337731589004.pdf

26. Fujii L; Clain E; Morris M; Levy J. Anterior spinal cord infarction with permanent paralysis following endoscopic ultrasound celiac plexus neurolysis. 2012; 44: E265-E266. doi: 10.1055/s-0032-1309708

27. Teixeria M; Neto E; Marinho J; Angelos J; Martìn M. Celiac plexus neurolysis for the treatment of upper abdominal cancer pain. Neuropsychiatric Disease and
Treatment. 2013:9 1209-1212. doi: 10.2147/NDT. S43730

28. Viùdez A; Miranda C; Arias Fernando; et all. Situación actual en el tratamiento del cáncer gástrico. Rev Esp Enferm Dig. 2012 Mar 104(3). http://dx.doi. org/10.4321/S1130-01082012000300006

29. Wong GY, Schroeder DR, Carns PE, Wilson JL, Martin DP, Kinney MO, Mantilla CB, Warner DO. Effect of neurolytic celiac plexus block on pain relief, quality of life, and survival in patients with unresectable pancreatic cancer: a randomized controlled trial. JAMA. 2004 Mar 3;291(9):1092-9. DOI: 10.1001/ jama.291.9.1092

30. Staats PS, Hekmat H, Sauter P, Lillemoe K. The effects of alcohol celiac plexus block, pain, and mood on longevity in patients with unresectable pancreatic cancer: a double-blind, randomized, placebocontrolled study. Pain Med. 2001 Mar;2(1):28-34. DOI: 10.1046/j.1526-4637.2001.002001028.x

31. Birthi P, Sloan P. Interventional treatment of refractory cancer pain. Cancer J 2013 Sep-Oct;19(5):390-396. DOI: 10.1097/PPO.0b013e3182a631a2 